\title{
AminoxyTMT: A novel multi-functional reagent for characterization of protein carbonylation
}

\author{
Somaieh Afiuni-Zadeh ${ }^{1,2}$, John C. Rogers ${ }^{3}$, Sergei I. Snovida ${ }^{3}$, Ryan D. Bomgarden³, and \\ Timothy J. Griffin² \\ ${ }^{1}$ Department of Laboratory Medicine and Pathology, University of Minnesota, Minneapolis, \\ MN, ${ }^{2}$ Department of Biochemistry, Molecular Biology and Biophysics, University of Minnesota, \\ Minneapolis, MN, and ${ }^{3}$ Thermo Fisher Scientific, Rockford, IL
}

BioTechniques 60:186-196 (April 2016) doi 10.2144/000114402

Keywords: Post translational modification, Protein carbonylation, Immunoblotting, Mass spectrometry, Tandem Mass Tag, AminoxyTMT

Supplementary material for this article is available at www.BioTechniques.com/article/114402.

Protein carbonylation is a common oxidative stress (OS)-driven post-translational modification (PTM). Proteome-wide carbonylation events can best be characterized using a combination of analytical approaches. Immunoblotting of carbonylated proteins provides data on the extent of modifications within complex samples, as well as a broad comparison of carbonylation profiles between different biological states (e.g., disease versus control), while mass spectrometry (MS)-based analysis provides information on proteins susceptible to carbonylation, as well as the potential for quantitative characterization of specific sites of amino acid modification. Here, we present a novel use for aminoxyTMT, a derivative of the Tandem Mass Tag (TMT) isobaric labeling reagent, which utilizes an aminooxy functional group for covalent labeling of reactive carbonyls in proteins. When coupled with anti-TMT antibody, we demonstrate the use of aminoxyTMT for immunoblot profiling of protein carbonylation in complex mixtures, as well as enrichment of modified peptides from these mixtures. Proof-of-principle experiments also show the amenability of aminoxyTMT-labeled carbonylated peptides enriched from complex mixtures to identification using tandem MS (MS/MS) and database searching, as well as quantitative analysis using TMT-based reporter ion intensity measurements.

Protein carbonylation is a form of oxidation promoted by reactive oxygen species (ROS). Under oxidative stress conditions where ROS are in excess, covalent and irreversible introduction of carbonyl groups into proteins is a prominent post-translational modification (PTM) (1-3). Protein carbonylation can occur by several mechanisms, but the two main contributors are: (i) direct metal catalyzed oxidation (MCO) of specific amino acid chains (lysine, arginine, and proline) resulting in $\alpha$-aminoadipic semialdehyde (AAS) and $\gamma$-glutamic semialdehyde (GGS) respectively (4-7); and (ii) secondary reactions of nucleo- philic amino acid side chains (sulfhydryl group of cysteine, $\varepsilon$-amino group of lysine, and imidazole group of histidine) alkylated by ROS-induced lipid peroxidation products (8-10). These products include acrolein (ACR), malondialdehyde (MDA), 4-hydroxy-2-nonenal (HNE), and 4-oxo-trans-2,3-nonenal (4-ONE) via both Schiff base and/or Michael addition (11). Carbonylation is an accepted biomarker of oxidative stress $(1,2,12)$ and is associated with several pathological states, including alteration of metabolism and induction of apoptosis and cell death $(12,13)$, neurodegenerative disease such as Parkin- son's and Alzheimer's (14,15), rheumatoid arthritis (16), aging (17), diabetes, and obesity (18).

A number of analytical approaches have been developed to study proteomewide protein carbonylation with immunoblotting-based approaches being some of the most common. Here, probes capable of forming covalent bonds (e.g., through Schiff base formation with a hydrazide) with reactive carbonyls, primarily aldehydes, are added to a protein mixture. Once tagged, proteins are separated using SDS-PAGE and detected by immunoblotting using antibodies recognizing

\section{METHOD SUMMARY}

The aminoxyTMT reagent allows quantitative immunoblotting of proteins carrying reactive carbonyls. A derivative of the Tandem Mass Tag (TMT) reagents, it is amenable to tandem mass spectrometry (MS/MS) analysis, enabling identification of sites of amino acid carbonylation. Using the available anti-TMT antibody resin, aminoxyTMT-labeled peptides from complex mixtures can be enriched and then characterized using MS/MS. Finally, using the isobaric tagging features of the TMT reagent, aminoxyTMT can be employed for quantitative analysis of protein carbonylation, with the potential for multiplexed comparisons across many conditions. 
the probe label. Dinitrophenylhydrazine (DNPH) is one commonly used probe in this approach (19). Such immunoblottingbased approaches provide a visual profile of protein carbonylation within a complex protein mixture, enabling global comparisons of protein carbonylation between different samples (e.g., healthy versus disease samples). However, immunoblotting alone does not provide a complete picture of protein carbonylation, as the technique lacks the ability to directly identify proteins susceptible to modification, ascertain the amino acid site of carbonylation, or characterize the types of carbonyl modification (e.g., MCO-based modifications, HNE).

Mass spectrometry (MS)-based approaches complement immunoblotting techniques. Using tandem mass spectrometry (MS/MS) coupled with sequence database searching, identities of proteins susceptible to carbonylation, as well as the sites and types of modification, can be revealed (11). Generally, MS-based approaches for characterizing carbonylation in complex protein mixtures employ an enrichment step to isolate relatively low abundance, modified proteins and/or peptides prior to MS analysis. A number of MS-based approaches employing enrichment have been described for protein carbonylation analysis (20-22). Some of these approaches have also employed carbonyl-reactive probes that incorporate stable isotopes to enable relative quantification of carbonyl modification states $(20,21,23)$.

Several of the approaches described above employ multi-functional reagents, which allow the use of complementary methods for characterizing protein carbonylation. Most notable are biotinbased carbonyl-reactive probes that offer immunoblotting capabilities using avidin/ streptavidin derivatives for detecting and visualizing proteins containing carbonyl modifications. The avidin-biotin interaction is also useful for affinity enrichment of labeled proteins or peptides from complex mixtures, followed by MS/MS analysis. Despite some challenges due to fragmentation of the relatively large carbon linkers used in biotin-based reagents complicating MS/MS spectra interpretation (24), success has been reported in identifying amino acid sites of modification in peptides using MS/MS and sequence database searching $(21,22,25)$. In some cases, the biotin-based probes have been synthesized with either normal or heavy stable isotopes to enable relative quantification (20).

Here we describe a new multi-functional reagent for protein carbonylation analysis. The reagent, aminoxyTMT, is a derivative of the popular, MS/MS-compatible Tandem Mass Tag (TMT) isobaric labeling reagents from Thermo Fisher Scientific that contains a carbonyl-reactive aminooxy group $(26,27)$. Focusing on the common carbonyl modification by HNE on protein side-chains, we demonstrate the effectiveness of aminoxyTMT for both immunoblotting to visualize protein carbonylation using the available anti-TMT antibody and enriching aminoxyTMT-labeled peptides from complex mixtures. The TMT-based probe also enables identification of amino acid sites of modification by MS/MS and sequence database searching. Finally, we demonstrate how the isobaric aminoxyTMT tag can be used for relative quantification of carbonylation state, with the potential for high-level multiplexing.

\section{Material and methods}

\section{Reagents}

The following reagents were used in this study: BSA (Sigma Aldrich Corp., St. Louis, MO), tris(2-carboxyethyl) phosphine(TCEP) (Thermo Fisher Scientific, Rockford, IL), 4-hydroxynonenal (HNE) (Cayman Chemical Company, Ann Arbor, MI), triethylammonium acetate (TEAA) (Sigma Aldrich Corp.), (N-morpholino)ethanesulfonic acid (MES) (Sigma Aldrich Corp.), anti-HNE antibody (EMD Millipore, Darmstadt, Germany), anti-TMT antibody (Thermo Fisher Scientific), anti-TMT resin (Thermo Fisher Scientific), N,N-Diisopropylethylamine (DIPEA) (Sigma Aldrich Corp.), triethylammonium bicarbonate (TEAB) (Sigma Aldrich Corp.), aminoxyTMT126 and aminoxyTMT131 (Thermo Fisher Scientific).

\section{Animal protocol}

Male wild-type C57Bl/6J mice were weaned at 3 weeks of age and then fed high fat chow (20\% protein, 35.5\% fat, $36.3 \%$ carbohydrate by weight, $60 \%$ fat by calories) (BioServlndustries, Frenchtown, $\mathrm{NJ}$ ) or standard chow (18.6\% protein, $6 \%$ fat, $44 \%$ carbohydrate by weight) (Teklad Global, Madison WI). Ob/ob mice were purchased at 10 weeks of age and maintained for 2 weeks on a normal chow diet before sacrifice. At 12 weeks of age (9 weeks on a high-fat chow or normal chow diet), animals were sacrificed, and liver tissue was removed and flash-frozen in liquid nitrogen. Frozen tissue was stored at $-80^{\circ} \mathrm{C}$ before analysis. The University of Minnesota Institutional Animal Care and Use Committee approved all animal procedures.

\section{Preparation of in vitro}

HNE modified proteins

One-hundred micrograms of BSA as standard protein as well as $1 \mathrm{mg}$ mouse liver mitochondria lysate at a concentration of $10 \mathrm{mg} / \mathrm{mL}$ were used in these studies. A crude, mitochondria-enriched cellular fraction was prepared from frozen liver tissue as previously described (28). Both BSA (2 mg/ml) and solubilized mouse mitochondrial proteins were reduced with $100 \mathrm{mM}$ TCEP and treated with HNE for 2 $h$ at $37^{\circ} \mathrm{C}$ in $100 \mathrm{mM}$ TEAA buffer, pH 7.2.

AminoxyTMT labeling of carbonylated proteins

BSA modified with HNE at cysteine, histidine and lysine (see Figure $1 \mathrm{~A}$ ) and mouse liver mitochondria lysate samples were acetone precipitated to remove excess unreacted HNE, and then $10 \mu \mathrm{l} 4$ $\mathrm{M}$ aniline was added to $1 \mathrm{ml}$ of the sample (for a final aniline concentration of $4 \mathrm{mM}$ ) to facilitate the reaction. The sample was labeled with $1 \mathrm{mM}$ aminoxyTMT126 in MES buffer, pH 5.0 for $2 \mathrm{~h}$ at room temperature, forming stable oxime bonds between the HNE aldehyde and the aminoxyTMT reagent (see Figure 1B). Labeled protein was then alkylated with iodoacetamide and digested with trypsin overnight (1:50 protease to substrate) using the filter-aided sample preparation (FASP) method (29), followed by clean-up using solid-phase extraction and subsequent storage at $-20^{\circ} \mathrm{C}$ for future experiments. A liver mitochondria lysate sample was also labeled with aminoxyTMT using this same method in an attempt to detect endogenous carbonylation events.

\section{Immunoblot analysis}

Protein samples were divided into aliquots and solubilized in gel loading sample buffer [0.0625 M Tris, pH 6.8; 1\% SDS (w/v); 0.05\% bromophenol blue (w/v); 10\% glycerol (w/v); $1 \% \beta$-mercaptoethanol (v/v)]. Proteins were resolved by SDS-PAGE using a 10\% gel (Bio-Rad, Hercules, CA) and transferred to an Immobilion-FL 
(A)

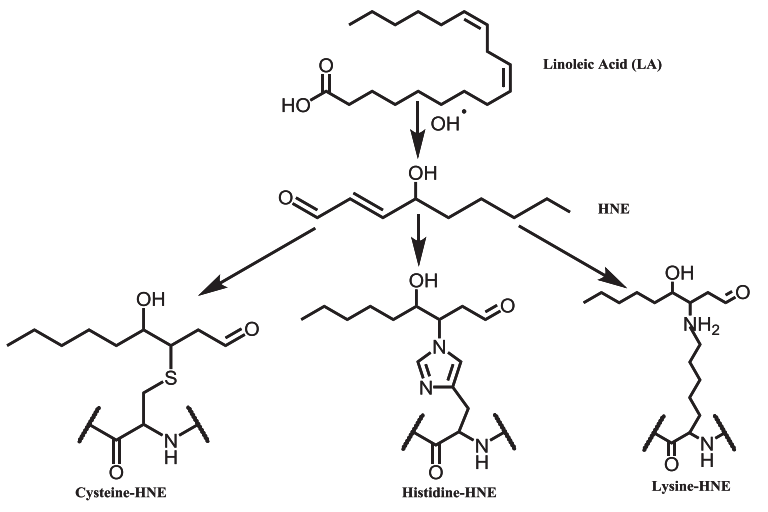

(B)

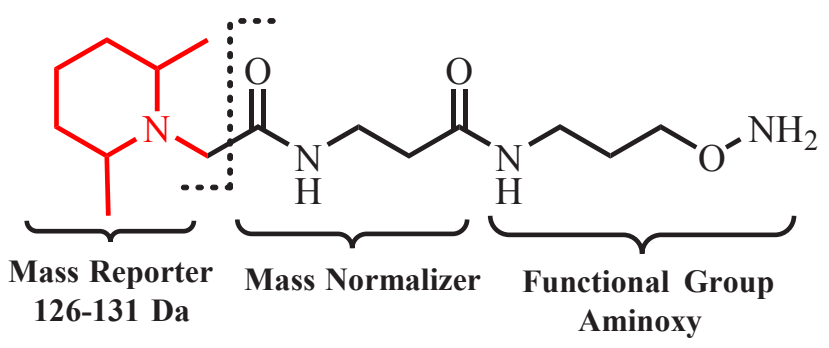

$\underbrace{\prod_{\mathrm{O}}^{N}}_{\downarrow_{\mathrm{TMT}}}$<smiles>CCCCCC(O)[C@@H](Cc1ccccc1)Cc1cccnn1</smiles>

Figure 1. Illustration of 4-hydroxynonenal (HNE) modifications on nucleophilic amino acid side chains introducing reactive carbonyl groups and aminoxy tandem mass tag (aminoxyTMT) structure. (A) HNE modifications of amino acid side chains. (B) Structure of aminoxyTMT reagent.

membrane (Pall Corporation, Pensacola, FL) in transfer buffer (12.5\% methanol, $25 \mathrm{mM}$ Tris base, $192 \mathrm{mM}$ glycine, $\mathrm{pH}$ 8.0). Following transfer, the membrane was incubated in $50 \mathrm{mM}$ sodium borohydride dissolved in PBS for 30-60 min. Immunoblots were blocked with Li-Cor blocking solution (LI-COR Biosciences, Lincoln, NE), and then incubated with an affinity purified polyclonal goat antirabbit antibody for HNE detection. The immunoblot membrane was also incubated with a goat anti-mouse antibody for TMT detection. Gels were visualized using the Odyssey Clx imaging system (LI-COR Biosciences) and Li-Cor infrared fluorescence secondary antibodies using wavelength channels at $700 \mathrm{~nm}$ and 800 nm for HNE and TMT detection, respectively.

\section{Enrichment of aminoxyTMT-labeled peptides}

Labeled peptides were passed through passed through an affinity chromatography anti-TMT resin for enrichment. The optimized enrichment procedure comprised the following steps: (i) Wash with 3 column volumes of TBS; (ii) Wash with 3 column volumes of $4 \mathrm{M}$ urea; (iii) Elute the sample with 3 column volumes of $10 \mathrm{mM}$ DIPEA in $500 \mathrm{mM}$ TEAB, pH 8.5. The TEAB acts as a competitive elution agent to the TMT tag. Eluted peptides were pooled and dried via vacuum centrif- ugation. Purified peptides were further processed by C18 stage-tip.

AminoxyTMT labeling of in vitro carbonylated proteins from obese and lean mice

Liver mitochondria lysates $(1 \mathrm{mg})$ from obese and lean mice were treated with HNE and labeled with aminoxyTMT126 and aminoxyTMT131, respectively. Proteins were digested using the FASP method and passed through the anti-TMT resin. Equal amounts of peptides were mixed and analyzed by LC-MS as described below.

LC-MS/MS analysis and sequence database searching for protein identification

For each sample analyzed, peptides were dissolved in $5 \mu \mathrm{L}$ water:acetonitrile:formic acid (98:2:0.01). Peptides were loaded directly onto a $13-\mathrm{cm}$ long $\times 75-\mu \mathrm{m}$ internal diameter fused silica PicoFrit (New Objective, Woburn, MA) capillary column packed in-house with Magic C18AQ resin (5 um, $200 \AA$ pore size; MichromBioResources, Auburn, CA) using an Eksigent 1D LC nanoflow system (Dublin, Ireland) and a MicroAS autosampler (Thermo Fisher Scientific). Peptides were eluted using a gradient of $10 \%-40 \%$ acetonitrile in $0.01 \%$ formic acid over $60 \mathrm{~min}$ at $320 \mathrm{nl} / \mathrm{min}$. An LTQ Orbitrap Velos mass spectrometer (Thermo Fisher Scientific) was used for MS detection. The electrospray voltage was set at $1.7 \mathrm{kV}$, and the heated capillary was maintained at $260^{\circ} \mathrm{C}$. The orbital trap was set to acquire survey mass spectra $(300-1800 \mathrm{~m} / \mathrm{z})$ with a resolution of 30,000 at $400 \mathrm{~m} / \mathrm{z}$ with automatic gain control (AGC) $1 \times 10^{6}$, maximum injection time of $10 \mathrm{~ms}$. The 6 most intense ions from the full scan were selected for fragmentation by higher-energy collisional dissociation with normalized collision energy 40 , activation time 0.1 , and detector settings of 15,000 resolution, AGC $5 \times 10^{4}$ ions, 100 ms maximum injection time, and FT first mass mode fixed at $111 \mathrm{~m} / \mathrm{z}$. Dynamic exclusion was set to a maximum 50 entries with a maximum retention period of $30 \mathrm{~s}$ and mass window of 0.5-1 amu. Data were acquired using Xcalibur software (Thermo Fisher Scientific).

ProteinPilot (version 4.5 AB; Sciex, Framingham, MA) was used for peptide sequence matching to MS/MS data and protein identification. The Paragon algorithm and protein grouping (30) was used to remove redundant hits. MS/MS 
data were searched against a mouse Uniprot database (version_042014) plus 115 common contaminant proteins (thegpm.org/crap/index). Reversed sequences of all proteins were appended to the forward sequences, for a total of 50,795 proteins in the concatenated database. The search parameters specified trypsin digestion, iodoacetamide for cysteine modification, and 90\% confidence for the matched peptide sequences, with the corresponding protein false positive discovery rate $<1 \%$. HNE and HNE-amnioxyTMT mass shifts for the amino acids histidine, lysine, and cysteine were 156.11 and 452.33 respectively.

\section{Results and discussion}

\section{Labeling of HNE-modified proteins}

Figure 1A shows HNE modifications on nucleophilic amino acid side chains that introduce reactive carbonyl groups, specifically aldehydes, into the protein structure. The aminoxyTMT tag (Figure 1B) consists of: (i) a reactive aminooxy group that allows covalent labeling of carbonylated proteins or peptides; (ii) a reporter group for relative quantification across all samples that can be detected after peptide fragmentation via MS/MS [note that each different TMT tag results in a reporter that increases in mass by one unit (e.g., between 126 and 131 Daltons for the 6-plex reagent)]; and (iii) a cleavable linker that includes a mass normalization group that balances mass differences from individual reporter fragments to keep the overall mass of the different tags constant (isobaric).

The aminoxy reactive group within the aminoxyTMT reagent has been utilized in many studies to promote specific labeling of reactive carbonyls, primarily aldehydes, in proteins $(21,31,32)$. It offers reactivity under mildly acidic or neutral conditions (pH 4.0-7.0) that promote protein stability (33). In terms of specificity in protein labeling, aminooxy groups are highly reactive to aldehydes and far less reactive to other carbonyls commonly found in proteins (e.g., those found in carboxylic acids or amides) or ketone groups. This behavior is observed even when using aniline as a catalyst (as we included in our protocol) (33). This specificity is a consequence of the strong electrophilic nature of the carbonyls found in aldehyde groups when compared with other groups, due to steric and electronic factors. The aminooxy forms a covalent oxime bond with the carbonyl (Figure 1B), which has been found to be more stable than the hydrazone bond formed by hydrazide reagents reacted with carbonyls (34). Due to this stability, we were able to eliminate the need for reduction with sodium borohydride, which is commonly used to stabilize hydrazone bonds (35).

\section{TMT channel}

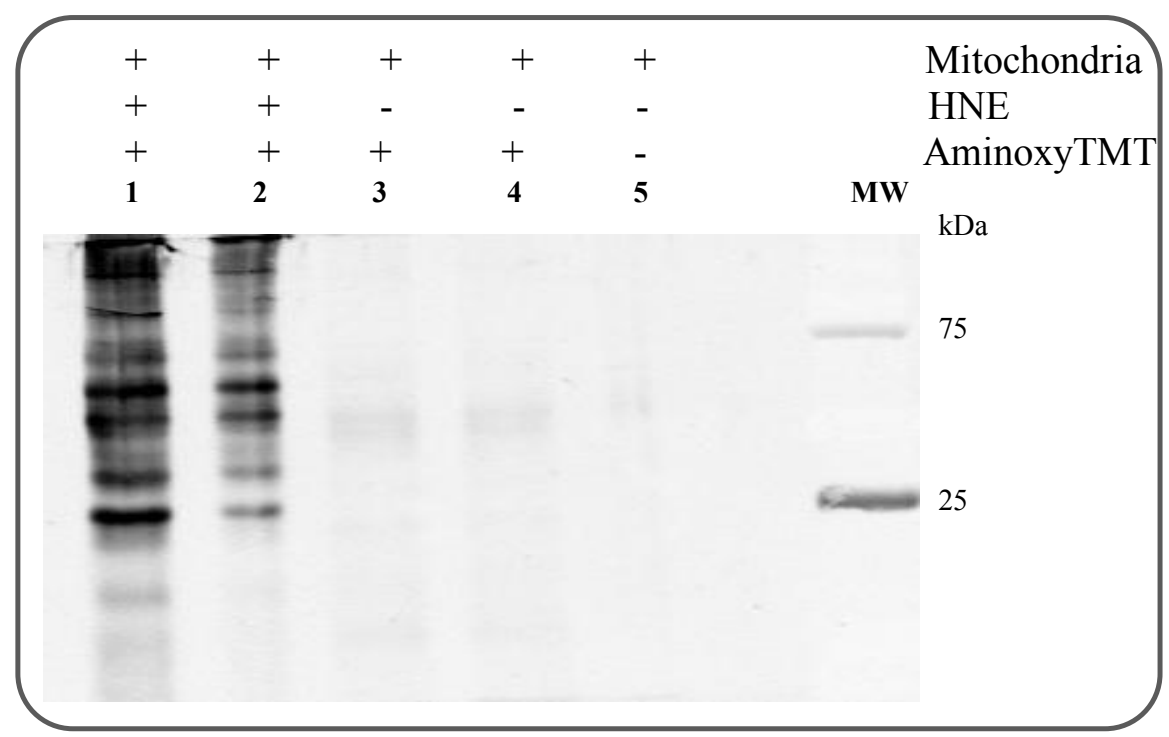

Figure 2. Immunoblot detection of enriched liver mitochondria lysate treated with 4-hydroxynonenal (HNE) and labeled with the aminoxy tandem mass tag (aminoxyTMT). The lane to the far right (labeled "MW") contains molecular weight markers, with the values of two of these markers indicated. In Lane 1, 6.5 $\mu \mathrm{g}$ of HNEmodified, aminoxyTMT-labeled protein was loaded, while Lane 2 was loaded with $3.3 \mu \mathrm{g}$ of the same sample. Lanes 3-5 were loaded with $3.3 \mu \mathrm{g}$ of total protein each from the same sample loaded in Lanes 1 and 2.

Table 1. HNE-modified BSA peptides labeled with aminoxyTMT with $99 \%$ confidence.

No. Modified peptides

1 SERMPCTTEDYLSLILN

2 YTRKVPQVSTPTLVEVSR

3 CTEDYLSLILNR

4 QFEKLLGEYGFQNALIVR

5 KNYQEAKDAFLGSFLYEYSR

6 EAKDAFLGSFLYEYSR

7 LPPLTADFAEDKDVCKKNYEAKDAFLGSFLYEYSR

The underlined amino acid in each peptide sequence is modified with 4-hydroxynonenal (HNE) and labeled with the aminoxy tandem mass tag (aminoxyTMT).

Immunoblot-based profiling

of protein carbonylation

The anti-TMT antibody enables selective recognition of proteins and peptides in complex samples that have been labeled with TMT-based reagents such as aminoxyTMT. To demonstrate the use of aminoxyTMT in specific labeling and visualization of carbonylated proteins in complex mixtures by immunoblotting, we analyzed both a single protein (BSA) and protein mixtures with HNE modifications. Results are shown using protein mixtures from mitochondria-enriched mouse liver samples treated with HNE, then labeled with aminoxyTMT and blotted using the anti-TMT antibody (Figure 2). Strong detection of the HNE-treated mitochondrial proteins is seen, with the signal scaling with amount of aminoxyTMTlabeled lysate added (Figure 2, first two lanes on the left of gel; lysate loading in the second lane was half that in the first lane). We did not directly quantify the signals in each lane, but relative amounts of labeled, carbonylated proteins could be accurately quantified via fluorescent measurements or densitometry, depending on the secondary antibody reporter used. Meanwhile, the lanes containing mitochondrial proteins that were not modified with HNE show only faint signal when labeled by aminoxyTMT and detected via immunoblotting. This faint signal is most likely due to low-levels of endogenous carbonylation of mitochondrial proteins, which has also been detected by others (36), although non-specific labeling of the proteins cannot be entirely ruled out. The increase in signal for the samples where proteins were known to carry reactive aldehyde groups due to their modification with HNE does, however, point to specific and strong reactivity of the aminoxyTMT reagent for carbonyl modifications in proteins. 

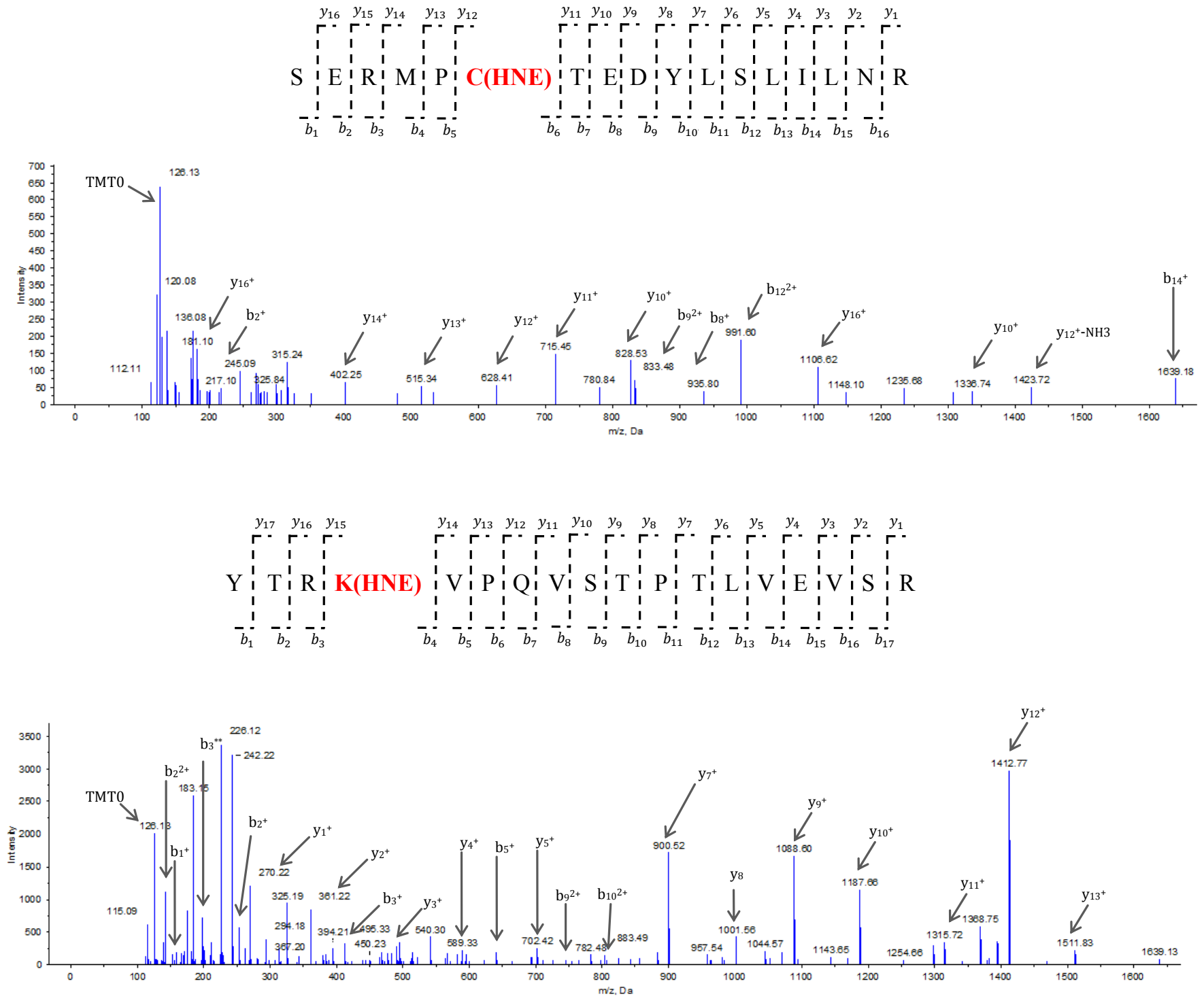

Figure 3. Representative peptide sequence matches of 4-hydroxynonenal (HNE)-modified, aminoxy tandem mass tag (aminoxyTMT)-labeled BSA peptides, one modified at a cysteine and the other at lysine. The reporter ion related to the TMT $(\mathrm{m} / \mathrm{z} \approx 126.12)$ is prominent in both tandem MS (MS/MS) spectra, along with sequence-specific b- and y-ions.

MS/MS analysis of carbonylated proteins

To demonstrate the amenability of aminoxyTMT-labeled carbonyls to MS/MS analysis and sequence database searching for identifying HNE-modified proteins and their modified sites, we focused on HNE-modified BSA. Mass spectrometry analysis of non-modified BSA as a control versus HNE-modified BSA is shown in Table 1. No HNE-aminoxyTMT modified peptides were identified in non-modified BSA, whereas seven aminoxyTMT-labeled peptides were detected with high confidence in the HNE-modified BSA. This demonstrates successful characterization of HNE-modified peptides labeled with aminoxyTMT via MS/MS. Represen- tative MS/MS spectra of HNE-modified, aminoxyTMT-labeled BSA peptides, one modified at a cysteine and the other at lysine, are shown in Figure 3. Notably, the reporter ion related to the TMT tag $(\mathrm{m} / \mathrm{z}$ $\approx 126.12$ ) is prominent in both $\mathrm{MS} / \mathrm{MS}$ spectra, along with sequence-specific band y-ions.

Affinity enrichment and MS/MS analysis: complex mixtures

Anti-TMT antibody-derivatized resin can be used for enrichment of aminoxyTMTlabeled peptides from complex mixtures prior to MS/MS analysis. This addresses one of the challenges in characterizing relatively low-abundance peptides containing amino acid sites susceptible to carbonylation within biological mixtures. Beyond its specificity in binding the TMT label, anti-TMT resin has a number of benefits as an affinity reagent. Mild conditions [10 mM N-N diisopropylethylamine (DIPEA) in 500 mM TEAB, pH 8 as elution buffer] can be used to competitively elute resin-bound peptides. These elution conditions minimize any undesired side-reactions with peptides or the resin. The TEAB buffer is also volatile and easily removed by vacuum centrifugation.

We explored the benefits of affinity enrichment for identification of aminoxyTMT-labeled, HNE modified peptides from enriched mitochondrial protein samples from mouse liver. Here, we identified 33 labeled, HNE-aminoxyTMT 
(A)

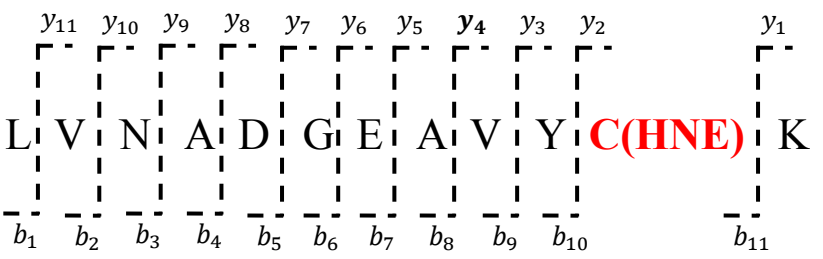

Catalase, sp|P24270|CATA_MOUSE

(B)

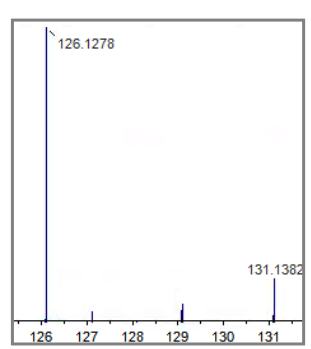

$\begin{array}{llll}y_{19} & y_{18} & y_{17} & y_{16}\end{array}$

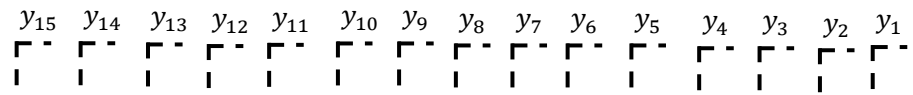

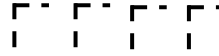

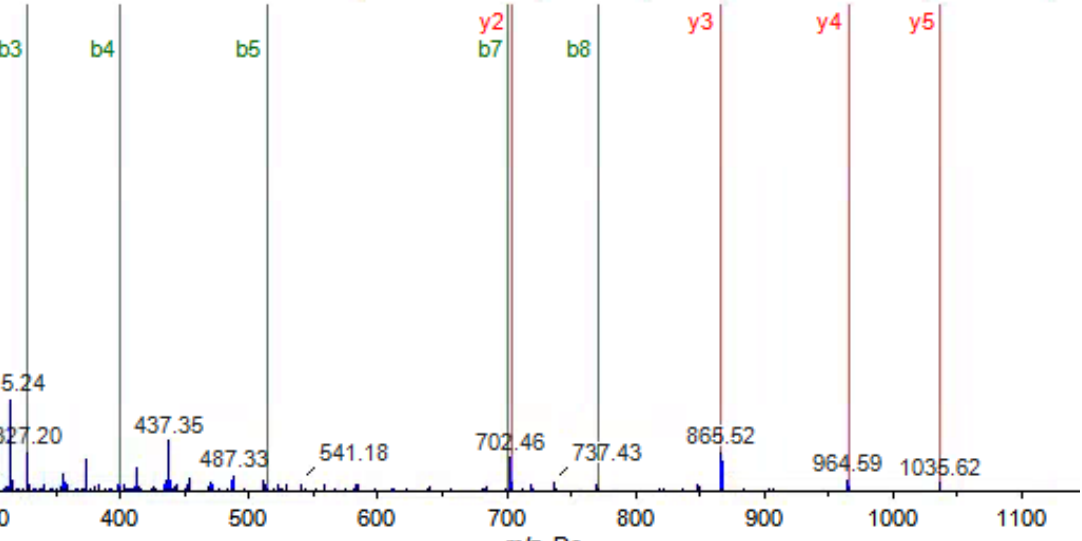

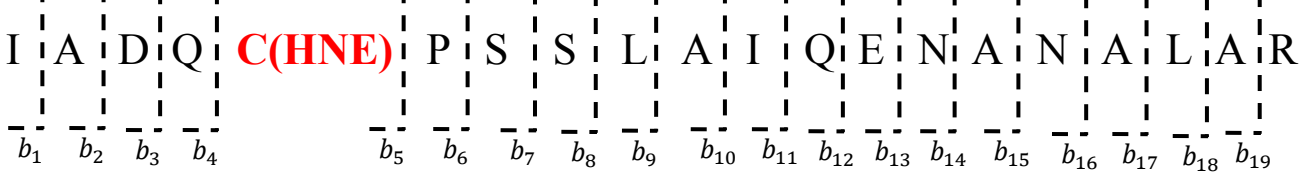

Fructose-bisphosphate aldolase, sp|Q91Y97|ALDOB MOUSE

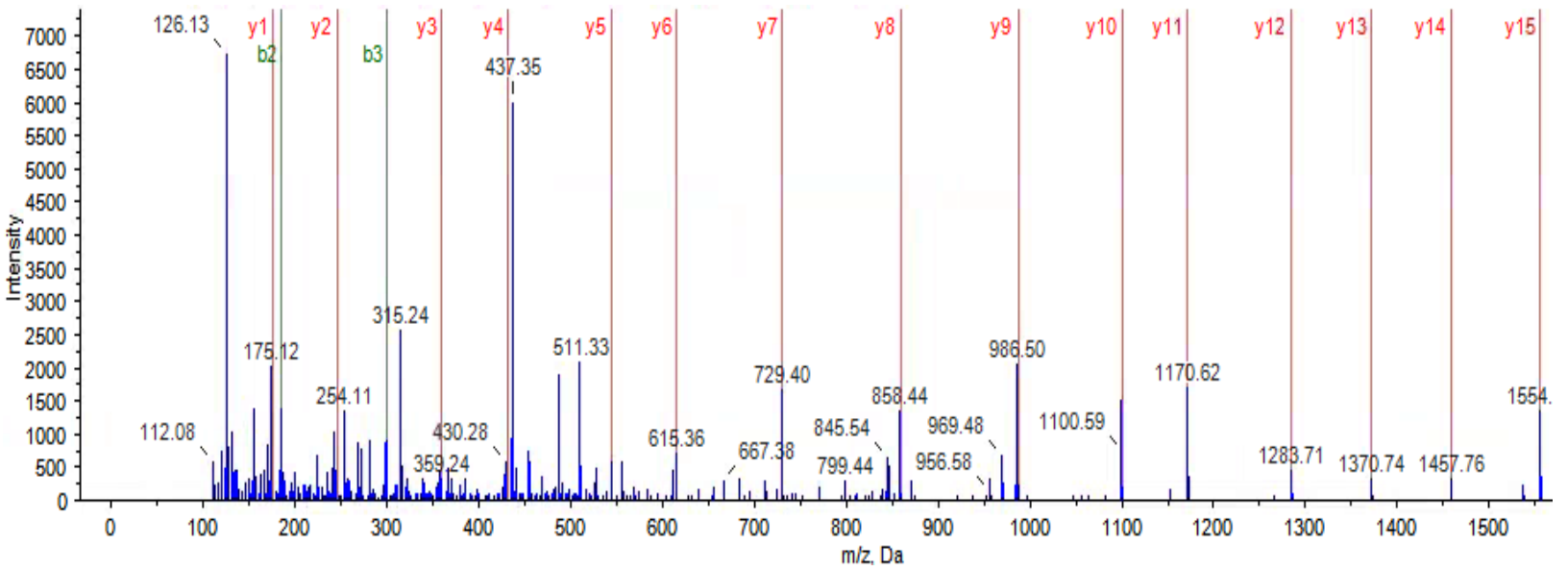


modified peptides before enrichment compared with 89 peptides carrying this modification after enrichment. Similar enrichment results were also observed for relatively simple samples, such as HNE-labeled BSA, and other complex samples (e.g., HNE-labeled proteins from adipose tissue; data not shown). Focusing on the results from crude mitochondria preparations from liver, the modified peptides identified after enrichment are listed in Supplementary Table S1. In addition to filtering by confidence scores assigned by ProteinPilot, all MS/ MS spectra were manually inspected to avoid false hits. All spectra show intense reporter ion at $126.12 \mathrm{~m} / \mathrm{z}$ and histidinemodified peptide peaks at 266.1, indicative of aminoxyTMT tag loss and detection of the Histidine-HNE immonium ion during MS/MS fragmentation. As would be expected from the analysis of a crudely enriched mitochondrial sample, many peptides identified are derived from known mitochondrial proteins, along with some cytoplasmic proteins and proteins from other compartments such as peroxisomes. Annotated spectra of peptides listed in Supplementary Table S1 are shown in Supplementary Figure S1.

Based on sequence database searching, we found approximately $12 \%$ of identified peptides following enrichment were not HNE-TMT labeled peptides, indicating enrichment is not $100 \%$. However, almost three times more HNETMT modified peptides were identified when incorporating the enrichment step, demonstrating the value of enrichment using anti-TMT antibody.

Comparative analysis of TMT-labeled peptides in a complex mixture

The aminoxyTMT reagent also enables relative quantification of carbonylation state using isobaric peptide tagging and reporter ions detected by MS/MS. To demonstrate this functionality, we carried out a pilot experiment, labeling a portion of proteins with HNE from crudely enriched liver mitochondria obtained from either lean or obese mice. We chose these samples to demonstrate the utility of aminoxyTMT in quantitative analysis. We assumed lower anti-oxidant activity due to obesity (37) could result in differences in susceptibility of specific proteins to HNE modification, and/or abundance differences (due to altered expression or turnover) of proteins that are targets of this modification. The separate protein samples were labeled with aminoxyTMT reagents synthesized with either a 126 $\mathrm{m} / \mathrm{z}$ reporter ion (aminoxyTMT-126, obese mice) or aminoxyTMT carrying a $131 \mathrm{~m} / \mathrm{z}$ reporter ion (aminoxyTMT-131, lean mice).

Figure 4 shows data from two representative, high confidence peptides that showed large quantitative differences between obese ( $126 \mathrm{~m} / \mathrm{z}$ reporter ion) and lean (131 m/z reporter ion) mice, indicating increased abundance of the HNE modified peptide in obese mice compared with lean mice. It is unclear whether this difference is due to an overall increase in the abundances of these proteins in obese animals or a difference in susceptibility to HNE modification. However, there is some evidence that a high fat diet leading to obesity induces expression of both catalase (38) and fructose-bisphosphate aldolase (39), which could explain our results. Regardless, these samples provided a means to demonstrate the ability of the aminoxyTMT label to act as an isobaric tag for quantitative analysis of carbonylated peptides.

Our experimental results demonstrate the effectiveness of aminoxyTMT as a multi-functional reagent for protein carbonylation analysis. It is useful for immunoblotting to visualize protein carbonylation state in complex samples; using the anti-TMT resin, aminoxyTMT-labeled peptides can be enriched from complex mixtures prior to MS analysis. Although not demonstrated here, the immobilized anti-TMT antibody resin should also be capable of enrichment of aminoxyTMTlabeled proteins. The aminoxyTMT reagent is amenable to MS/MS analysis because it is based on the TMT-tag chemistry designed specifically for MS/MS applications, unlike other reagents (e.g., biotin hydrazide), which may exhibit fragmentation that interferes with interpretation of peptide fragmentation spectra (24). Finally, the aminoxyTMT reagent offers relative quantification of modified peptides utilizing the isobaric tagging functionality engineered into the TMT tag, with potential for high level multiplexing for comparisons across multiple conditions (e.g., time points, aging studies, etc.).

Despite its upside, some challenges still exist when using aminoxyTMT, including identification of low-abundance endogenous carbonyl modifications. We were not able to identify modified peptides with high confidence from samples not treated exogenously with HNE. The solution to this problem may lie in significant scale-up of starting material (e.g., tens of milligrams total protein) where possible. The aminoxyTMT label and affinity enrichment should be amenable to such a scale-up to more comprehensively identify and quantify endogenous carbonyl modifications.

Finally, we only concentrated on the prominently studied HNE carbonyl PTM. In principle, any modification that introduces a reactive carbonyl group into a protein (e.g., MCO) should be compatible with the aminoxyTMT reagent. However, a more focused study targeting other types of carbonyl modifications is needed to conclusively demonstrate the amenability of aminoxyTMT to characterize these PTMs. We attempted to identify endogenous carbonyl modifications (without HNE treatment) from enriched liver mitochondria. After labeling with aminoxyTMT and enrichment, we did identify some peptides modified with HNE, as well as a few MCO modified peptides, such as AAS-Lysine, and GGS-Proline. However, these matches were of poor quality compared with those obtained for HNE-treated samples, based both on assigned confidence scores and manual inspection of the results (data not shown). These data are consistent with the low abundance of endogenous modifications we observed in the immunoblotting results (Figure 2).

\section{Author contributions}

S.A. performed sample preparation, developed protocols, performed data analysis, and drafted the manuscript. J.C.R., S.I.S., and R.D.B. conceived of the study and participated in reagent preparation. T.J.G. conceived of the study, participated in the design and oversight of the study, and helped to draft and write the manuscript. All authors read, edited, and approved the final manuscript.

\section{Acknowledgments}

We thank members of the Center for Mass Spectrometry and Proteomics for support and instrumental analysis. We thank Pratik Jagtap and the Minnesota Supercomputing Institute for support and mainte- 
nance of software used in this study. We also thank members of the Bernlohr lab at the University of Minnesota for help with mouse samples and processing. This work was supported in part by $\mathrm{NIH}$ grant R01 DK084669 to T.J.G. This paper is subject to the NIH Public Access Policy.

\section{Competing interests}

The authors declare no competing interests.

\section{References}

1. Dalle-Donne, I., R. Rossi, D. Giustarini, A. Milzani, and R. Colombo. 2003. Protein carbonyl groups as biomarkers of oxidative stress. Clin. Chim. Acta 329:23-38.

2. Dalle-Donne, I., D. Giustarini, R. Colombo, R. Rossi, and A. Milzani. 2003. Protein carbonylation in human diseases. Trends Mol. Med. 9:169-176.

3. Soreghan, B.A., F. Yang, S.N. Thomas, J. Hsu, and A.J. Yang. 2003. High-throughput proteomicbased identification of oxidatively induced protein carbonylation in mouse brain. Pharm. Res. 20:17131720

4. Stadtman, E.R. and C.N. Oliver. 1991. Metalcatalyzed oxidation of proteins. Physiological consequences. J. Biol. Chem. 266:2005-2008.

5. Stadtman, E.R. and B.S. Berlett. 1997. Reactive oxygen-mediated protein oxidation in aging and disease. Chem. Res. Toxicol. 10:485-494.
6. Requena, J.R., C.C. Chao, R.L. Levine, and E.R. Stadtman. 2001. Glutamic and aminoadipic semialdehydes are the main carbonyl products of metalcatalyzed oxidation of proteins. Proc. Natl. Acad. Sci. USA 98:69-74.

7. Afiuni-Zadeh, S., X. Guo, G. Azimi, and E. Lankmayr. 2011. Optimization and application of microwave-assisted acid hydrolysis for rapid quantification of protein oxidation markers using LC-MS. Talanta 85:1835-1841.

8. Carini, M., G. Aldini, and R.M. Facino. 2004. Mass spectrometry for detection of 4-hydroxy-trans-2nonenal (HNE) adducts with peptides and proteins. Mass Spectrom. Rev. 23:281-305.

9. Davies, M.J., S. Fu, H. Wang, and R.T. Dean. 1999. Stable markers of oxidant damage to proteins and their application in the study of human disease. Free Radic. Biol. Med. 27:1151-1163.

10. Doorn, J.A. and D.R. Petersen. 2002. Covalent modification of amino acid nucleophiles by the lipid peroxidation products 4-hydroxy-2-nonenal and 4-oxo-2-nonenal. Chem. Res. Toxicol. 15:1445-1450.

11. Grimsrud, P.A., H. Xie, T.J. Griffin, and D.A. Bernlohr. 2008. Oxidative stress and covalent modification of protein with bioactive aldehydes. J. Biol. Chem. 283:21837-21841.

12. Furukawa, S., T. Fujita, M. Shimabukuro, M. Iwaki, Y. Yamada, Y. Nakajima, O. Nakayama, M. Makishima, et al. 2004. Increased oxidative stress in obesity and its impact on metabolic syndrome. J. Clin. Invest. 114:1752-1761.

13. Houstis, N., E.D. Rosen, and E.S. Lander. 2006. Reactive oxygen species have a causal role in multiple forms of insulin resistance. Nature 440:944-948.
14. Choi, J., H.D. Rees, S.T. Weintraub, A.I. Levey, L.S. Chin, and L. Li. 2005. Oxidative modifications and aggregation of $\mathrm{Cu}, \mathrm{Zn}$-superoxide dismutase associated with Alzheimer and Parkinson diseases. J. Biol. Chem. 280:11648-11655.

15. Choi, J., C.A. Malakowsky, J.M. Talent, C.C. Conrad, C.A. Carroll, S.T. Weintraub, and R.W. Gracy. 2003. Anti-apoptotic proteins are oxidized by Abeta25-35 in Alzheimer's fibroblasts. Biochim. Biophys. Acta 1637:135-141.

16. Mantle, D., G. Falkous, and D. Walker. 1999 Quantification of protease activities in synovial fluid from rheumatoid and osteoarthritis cases: comparison with antioxidant and free radical damage markers. Clin. Chim. Acta 284:45-58.

17. Levine, R.L. 2002. Carbonyl modified proteins in cellular regulations, aging and disease. Free Radic. Biol. Med. 32:790-796

18. Frohnert, B.I., A.R. Sinaiko, F.J. Serrot, R.E. Foncea, A. Moran, S. Ikramuddin, U. Choudry, and D.A. Bernlohr. 2011. Increased adipose protein carbonylation in human obesity. Obesity (Silver Spring) 19:1735-1741

19. Oliver, C.N., B.W. Ahn, E.J. Moerman, S. Goldstein, and E.R. Stadtman. 1987. Age-related Changes in Oxidized Proteins. J. Biol. Chem. 262:5488-5491.

20. Mirzaei, H. and F. Regnier. 2006. Identification and quantification of protein carbonylation using light and heavy isotope labeled Girard's P reagent. J. Chromatogr. A 1134:122-133.

21. Lee, S., N.L. Young, P.A. Whetstone, S.M. Cheal, W.H. Benner, C.B. Lebrilla, and C.F. Meares. 2006. Method to site-specifically identify and quantitate carbonyl end products

\section{X-MAN ${ }^{\circledR}$ Cell Lines}

Innovate

Impact

\section{Learn}

\section{Access Cutting Edge Genome Editing... For a fraction of the price of doing it yourself.}

- Leapfrog the often expensive and time consuming process of generating your own knockout lines. With X-MAN ${ }^{\otimes}$ Cell Lines, let Horizon generate the tools, reclaim tens of hours at the bench, and get back to your research.

- Over 2000 cell lines available today at a fraction of the price of doing it yourself

- Isogenic pairs of clonal mutant and wild type parental line provide the ideal control system

- Rapid haploid knockout on-demand generation service

- Successful proven pipeline for gene-editing cell lines 
of protein oxidation using oxidation-dependent element coded affinity tags (O-ECAT) and nanoliquid chromatography Fourier transform mass spectrometry. J. Proteome Res. 5:539547.

22. Chavez, J., J. Wu, B. Han, W.G. Chung, and C.S. Maier. 2006. New role for an old probe: affinity labeling of oxylipid protein conjugates by N'-aminooxymethylcarbonylhydrazino d-biotin. Anal. Chem. 78:6847-6854.

23. Han, B., J.F. Stevens, and C.S. Maier. 2007. Design, synthesis, and application of a hydrazide-functionalized isotope-coded affinity tag for the quantification of oxylipid-protein conjugates. Anal. Chem. 79:3342-3354.

24. Borisov, O.V., M.B. Goshe, T.P. Conrads, V.S. Rakov, T.D. Veenstra, and R.D. Smith. 2002. Low-energy collision-induced dissociation fragmentation analysis of cysteinyl-modified peptides. Anal. Chem. 74:2284-2292.

25. Han, D.K., J. Eng, H. Zhou, and R. Aebersold. 2001. Quantitative profiling of differentiationinduced microsomal proteins using isotopecoded affinity tags and mass spectrometry. Nat. Biotechnol. 19:946-951.

26. Chung, W.G., C.L. Miranda, and C.S. Maier. 2008. Detection of carbonyl-modified proteins in interfibrillar rat mitochondria using $\mathrm{N}$ '-aminooxymethylcarbonylhydrazino-D-biotin as an aldehyde/keto-reactive probe in combination with Western blot analysis and tandem mass spectrometry. Electrophoresis 29:1317-1324.

27. Slade, P.G., M.V. Williams, A. Chiang, E. Iffrig, S.R. Tannenbaum and J.S. Wishnok, 2011. A filtered database search algorithm for endogenous serum protein carbonyl modifications in a mouse model of inflammation. Mol Cell Proteomics 10: M111 007658.

28. Frezza, C., S. Cipolat, and L. Scorrano. 2007. Organelle isolation: functional mitochondria from mouse liver, muscle and cultured fibroblasts. Nat. Protoc. 2:287-295.

29. Wisniewski, J.R., A. Zougman, N. Nagaraj, and M. Mann. 2009. Universal sample preparation method for proteome analysis. Nat. Methods 6:359-362.

30. Shilov, I.V., S.L. Seymour, A.A. Patel, A. Loboda W.H. Tang, S.P. Keating, C.L. Hunter, L.M. Nuwaysir, and D.A. Schaeffer. 2007. The paragon algorithm, a next generation search engine that uses sequence temperature values and feature probabilities to identify peptides from tandem mass spectra. Mol. Cell. Proteomics 6:1638-1655.

31. Hahne, H., P. Neubert, K. Kuhn, C. Etienne, R. Bomgarden, J.C. Rogers, and B. Kuster. 2012. Carbonyl-reactive tandem mass tags for the proteome-wide quantification of $\mathrm{N}$-linked glycans. Anal. Chem. 84:3716-3724.

32. Zeng, Y., T.N. Ramya, A. Dirksen, P.E. Dawson, and J.C. Paulson. 2009. High-efficiency labeling of sialylated glycoproteins on living cells. Nat. Methods 6:207-209.

33. Wang, S., D. Gurav, O.P. Oommen, and O.P. Varghese. 2015. Insights into the mechanism and catalysis of oxime coupling chemistry at physiological pH. Chemistry 21:5980-5985.

34. Kalia, J. and R.T. Raines. 2008. Hydrolytic stability of hydrazones and oximes. Angew. Chem. Int. Ed. Engl. 47:7523-7526.

35. Meany D.L., H. Xie, L.V. Thompson, E.A. Arriaga, T.J. Griffin. 2007. Identification of carbonylated proteins from enriched rat skeletal muscle mitochondria using affinity chromatography-stable isotope labeling and tandem mass spectrometry. Proteomics 7:1150-1163.

36. Hagopian, K., R. Soo Hoo, J.A. LopezDominguez, and J.J. Ramsey. 2013. Calorie restriction influences key metabolic enzyme activities and markers of oxidative damage in distinct mouse liver mitochondrial sub-populations. Life Sci. 93:941-948.

37. Curtis, J.M., P.A. Grimsrud, W.S. Wright, X. Xu, R.E. Foncea, D.W. Graham, J.R. Brestoff, B.M. Wiczer, et al. 2010. Downregulation of adipose glutathione S-transferase A4 leads to increased protein carbonylation, oxidative stress, and mitochondrial dysfunction. Diabetes 59:1132-1142.

38. Rindler, P.M., S.M. Plafker, L.I. Szweda, and M. Kinter. 2013. High dietary fat selectively increases catalase expression within cardiac mitochondria. J. Biol. Chem. 288:1979-1990.

39. Nesteruk, M., E.E. Hennig, M. Mikula, J. Karczmarski, A. Dzwonek, K. Goryca, T. Rubel, A. Paziewska, et al. 2014. Mitochondrial-related proteomic changes during obesity and fasting in mice are greater in the liver than skeletal muscles. Funct. Integr. Genomics 14:245-259.

Received 31 December 2014; accepted 19 January 2016.

Address correspondence to Timothy J Griffin, Department of Biochemistry, Molecular Biology and Biophysics, University of Minnesota, Minneapolis, MN. E-mail: tgriffin@umn.edu

To purchase reprints of this article, contact: biotechniques@fosterprinting.com

\section{INNATE IMMUNITY}

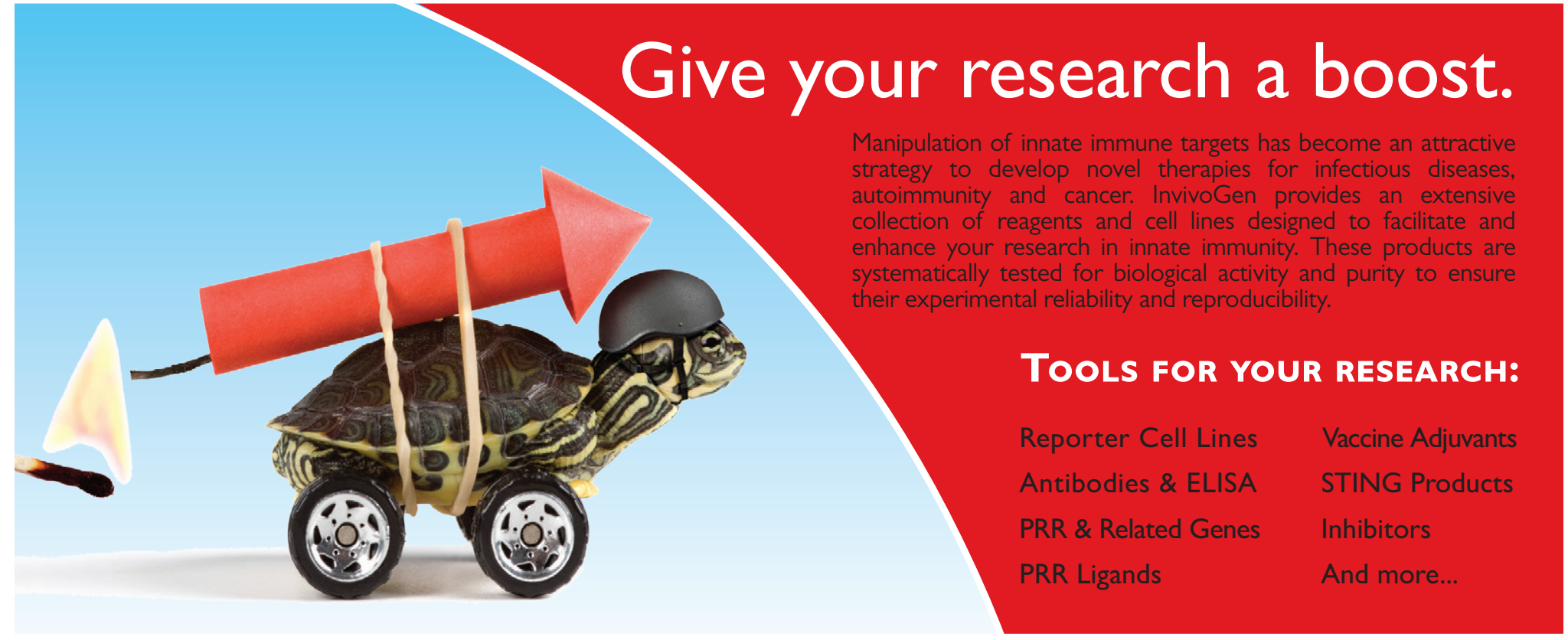

